\title{
Amphotericin-B and vancomycin-loaded chitosan nanofiber for antifungal and antibacterial application
}

\author{
Soroush Karimi', Pouran Moradipour ${ }^{1}$, Abbas Hemati Azandaryani', Elham Arkan ${ }^{1 *}$
}

${ }^{1}$ Nano Drug Delivery research center, Kermanshah University of Medical Sciences, Kermanshah, Iran

\begin{abstract}
In the present study, a mucoadhesive non-woven fiber mat $(\mathrm{d}=116 \mathrm{~nm})$ was fabricated by the electrospinning method using chitosan $(80 \% \mathrm{Wt})$, polyethylene oxide $(10 \% \mathrm{Wt})$, cysteine $(4 \% \mathrm{Wt})$ and drugs $(6 \% \mathrm{Wt})$, respectively. In addition, a comparative study was conducted to define effect of drugs and mucoadhesive agent on the nanofiber formation. FTIR, SEM, DSC and DMA were used to investigate the chemical and physical properties of the nanofibers. In vitro release of the drugs was assessed over a 48-hour period by the total immersion method. Release data were fitted to kinetic models, including the zero-order, first-order, Higuchi matrix, and Hixson-Crowell. Zone inhibition investigations were used to describe the inhibition content of vancomycin and amphotericin B loaded in the mats. The SEM images displayed a slight decrease in the fiber diameter with adding drugs and mucoadhesive agents. FTIR spectra confirmed that any undesirable reaction between VAN-AMB and CS-PEO was not observed. DSC test recognized the uniform distribution of drugs in the polymeric bead of the fiber without any crystal form. The elasticity modulus of the nanofiber was in an acceptable range for oral mucosa (approximately $5 \mathrm{Mpa}$ ). The results indicated that biodegradable mucoadhesive nanofibrous membranes released high concentrations of VAN in the first 24 hours, but the AMB release was affected in more controlled phenomena.
\end{abstract}

Keywords: Mucoadhesive. Vancomycin. Amphotericin-B. Electrospinning. Aphthous. Chitosan.

\section{INTRODUCTION}

Recurrent aphthous stomatitis (RAS) is the second prevalent disease of the oral mucosa causing painful and recurrent necrotic lesions in oral mucosa tongue and gums (Passaretti et al., 2001). Lacking exact and complete prophylactic therapy has led to development of several treats using various agents such as drugs, antibacterial, anti-acidic and antineoplastic materials (Chiu, Tsai, 2011). Histologically, RAS is caused by infiltration of mononuclear cells with fibrin coat (Ma et al., 2005). Although the lesions improve spontaneously after 10-14 days, specific treatment should be considered owing to severe pain and possible recreation (Costa-Pinto, Reis, Neves, 2011). Esters, chemical and physical trauma, use of cancer drugs, food allergy and infection exacerbate the indisposition (Brooks et al., 2013). Mucoadhesive drug delivery systems (MDDS) have been extensively

\footnotetext{
*Correspondence: E. Arkan. Nano Drug Delivery Research Center, Kermanshah University of Medical Sciences, Kermanshah, Iran. Tel./Fax: +98-833427493. E-mail: elhamarkan@yahoo.com iD
}

developed to treat oral diseases. In MDDS, drugs without first liver pass enter into the systemic circulation, which can lead to reduced dosage and side effects. Concerning antibiotics, it also can reduce antimicrobial resistance $(\mathrm{Lu}$ et al., 2012).

In this study, mucoadhesive nanofibers were loaded with amphotericin-B (AMB) as the curing agent of fungi and vancomycin (VAN) as a barrier agent. AMB is a poly-N antibiotic with a similar structure to nystatin. Poly-N molecules are considered amphipathic, since they contain hydrophilic and lipophilic fragments. These molecules bind to ergosterol (a specialized cell membrane sterols fungus) and create artificial pores. The drug is poorly absorbed from the gastrointestinal tract. Usually, it is injected in the form of a colloidal non-lipid suspension widely distributed in all tissues. The half-life of AMB is approximately 2 weeks excreted mainly via a slow hepatic metabolism. Its mechanism of action is due to its effect on permeability and transmission characteristics of fungal membranes (Hamill, 2013). Vancomycin is commonly utilized in treating gram-positive microorganism dangerous infections (such as methicillin-resistant 
of staphylococci). VAN is not absorbed from the gastrointestinal tract. In the injectable form diffuses into the most tissues where excretes unchanged through urine. The side effects of VAN include chills, fever, phlebitis and so forth. Dose and controlled release behavior of antibiotics and it is so important. Nanotechnology helps to manipulate physical and chemical properties of materials; therefore, the demand changes are applied on the DDS.

Nowadays, electrospinning as a versatile and flexible method is useful to make nanofibrous mats with the highest surface to volume ratio. Increasing the active surface reduces the dosage of the drugs. In electrospinning, high voltage is applied to create electrostatic forces overcoming viscosity and surface tension of the initial solution (a mixture of polymer, solvent, and drugs). After formation of the first jet, the solvent evaporates and solid nanofibers gather on the surface of collector (Li, Xia, 2004).

Recent research introduces a mucoadhesive patch of chitosan (CS), polyethylene oxide (PEO) and cysteine (Cys) loaded with VAN and AMB. In the present study, we used chitosan as a non-toxic, biocompatible and biodegradable polymer. The existence of amine groups gives a cationic property to the CS solutions, causing problems in the electrospinning process. (Li, Xia, 2004; Puppi et al., 2010). Anionic or nonionic polymers are usually used a modifier agent. PEO is a synthetic nontoxic, nonionic, hydrophilic, mucoadhesive and lubricant polymer (Ma, Deng, Chen, 2013; Cannon, Long, 2008; Fullana, Wnek, 2012).

\section{MATERIAL AND METHODS}

\section{Material}

Chitosan (CS) (low molecular weight, DD: 91.2\%, Viscosity: $220 \mathrm{mPa}$ ) was purchased from Hocheon Co. (China). Polyethylene oxide, Glacial acetic acid, acetone, absolute ethanol, $\mathrm{NaOH}, \mathrm{KH}_{2} \mathrm{PO}_{4}$, Vancomycin, Amphotericin-B and cellulose acetate membrane were obtained from Sigma Aldrich (USA).

\section{Electrospinning Procedures}

The spinal solutions were prepared as CS: PEO
(80: 20) wt\%, CS: PEO: Cys (80: 19: 1) wt \%, CS: PEO: VAN: AMB (80:10:5:5) wt $\%$, CS: PEO: VAN: AMB: Cys (80:10:4:4:2) wt $\%$ adding to the solvent mixture contain of acetic acid: water $(80: 20) \mathrm{wt} \%$. Each solution was stirred for $24 \mathrm{~h}$ at room temperature. Cysteine as a mucoadhesive agent was added to the above solution in different concentrations (1, 2 and $3 \% \mathrm{~W} / \mathrm{W}$ of polymers). Vancomycin was added to the above mixture in the $8 \%$ $\mathrm{W} / \mathrm{W}$ for drug loading. Herein, the electrospinning setup consisted of a syringe pump, a drum collector covered by an aluminum sheet and a high voltage supply. A plastic syringe (capacity $=1 \mathrm{~mL}$ ) was fuelled with CS/ PEO solution equipped with an 18-gauge needle. The tip of the needle was connected to the high voltage supply and placed in front of the drum collector with opposite charge. For electrospinning process, different parameters, including drum rotating speed (varied from 300 to 750 $\mathrm{rpm})$, volumetric flow rate $(0.5$ to $2.5 \mathrm{~mL} / \mathrm{h})$, distance between nozzle and drum (varied from 120 to $200 \mathrm{~mm}$ ), temperature $\left(20\right.$ to $\left.45^{\circ} \mathrm{C}\right)$ and applied voltage (12 to $22 \mathrm{kV}$ ) were investigated. Table I shows the obtained optimum parameter for electrospinning.

\section{Cross-linking of nanofiber}

To enhance mechanical properties of chitosan nanofibers, a $50 \% \mathrm{Wt}$ solution of glutaraldehyde (GTA) was used in water. $10 \mathrm{~mL}$ of this solution was poured in a petri dish and placed in vacuumed desiccator for $2 \mathrm{~h}$, then it moved to a vacuum oven with a temperature of $40{ }^{\circ} \mathrm{C}$ for $24 \mathrm{~h}$ to eliminate the unreacted GTA from the nanofiber mats (Hargreaves, Nguyen \& Ryan, 2006).

\section{Apparatus}

The electrospun nanofibers were characterized with SEM (Philips XL30 microscope), FTIR (Schimadzu IRprestige 21), DSC (Mettler Toledo STAR system, DSC821, Columbus, Ohio), and DMA (STM 50).

\section{In vitro release and kinetics study}

The immersion method was investigated in vitro release of VAN and AMB from nanofibrous membrane.

TABLE I - Optimum conditions for electrospinning

\begin{tabular}{ccccc}
\hline Temperature $\left[{ }^{\circ} \mathrm{C}\right]$ & $\begin{array}{c}\text { Distance spinneret } \\
\text { collector }[\mathrm{mm}]\end{array}$ & Voltage $[\mathrm{KV}]$ & $\begin{array}{c}\text { Solution flow } \\
\text { rate }[\mathrm{mL} / \mathrm{h}]\end{array}$ & $\begin{array}{c}\text { Concentration } \\
{\left[\%{ }^{\wedge} \mathrm{w} / \mathrm{t}\right]}\end{array}$ \\
\hline $38-40$ & 120 & $15-20$ & 1.2 & $2 / 7$ \\
\hline
\end{tabular}


Approximately $50 \mathrm{mg}$ of the electrospun fibers was placed in to the dialysis bag (SERVA, MWCO, $12000 \mathrm{Da}$ ), $2 \mathrm{~mL}$ PBS ( $\mathrm{pH}=7.4)$ was added to each bags; this part acted as the donor solution. The bag was immersed in 50 $\mathrm{mL}$ of receptor medium (phosphate buffer saline $0.2 \mathrm{M}$, $\mathrm{pH}$ 7.4) and incubated at $37^{\circ} \mathrm{C}$ under magnetic stirring (approximately $400 \mathrm{rpm}$ ). At specified time intervals, $1 \mathrm{~mL}$ of solution was taken and replaced with the same volume of fresh buffer. For drug concentration assay, the taken samples were analyzed at $283 \mathrm{~nm}$ and $416 \mathrm{~nm}$ for VAN and AMB using a UV spectrophotometer (UVmini-1240, Shimadzu, Germany) (Rostami, Kashanian, Azandaryani, 2014). The quantity of the drug released was calculated according to Equation 1:

$$
\mathrm{C}_{\mathrm{n}}=\frac{\mathrm{C}+\left(\mathrm{C}_{\mathrm{n}-1}\right) \mathrm{V}}{\mathrm{V}_{\text {total }}}
$$

where $\mathrm{C}_{\mathrm{n}}$ is the concentration of the released drug, $\mathrm{C}$ is the concentration in the withdraw sample, $C_{n-1}$ is the concentration of the drug in the $\mathrm{n}-1$ sample, $\mathrm{V}$ and $\mathrm{V}_{\mathrm{t}}$ are the picked up and the total volume of receptor solution, respectively. The release results are plotted as the cumulative percentage of drug content in solution $v s$ time.

For in vitro study of release kinetics, several approaches can be used such as analysis of variance, model-independent, and model-dependent approaches. In this study, model-dependent approaches were used to compare dissolution profiles. In model-dependent approaches, release data were fitted to kinetic models, including the zero-order (Eq. 2), first-order (Eq. 3), Higuchi matrix (Eq. 4), Hixson-Crowell (Eq. 5) release equations to find the equation with the best fit (Rostami, Kashanian, Azandaryani, 2014; Venkateswarlu, Manjunath, 2004).

$$
\begin{gathered}
\mathrm{C}=\mathrm{K}_{0} \mathrm{t} \\
\log \mathrm{C}=\log \mathrm{C}_{0}-\mathrm{Kt} / 2.303 \\
\mathrm{Q}=\mathrm{Kt}^{1 / 2} \\
\sqrt[3]{W_{0}}-\sqrt[3]{W_{t}}=K_{s} t
\end{gathered}
$$

\section{Antibacterial properties characterization}

The antibacterial activities of CS/PEO nanofibrous layers with and without VAN/AMB were investigated in the presence of gram-positive streptococcus (Strep.) by the zone inhibition method. An $8 \mathrm{~mm}$ diameter circular was cut from each mat and sterilized by placing under UV radiation for $20 \mathrm{~min}$. Then, they were transferred to bacterium suspension then incubated for 24 hours at $37^{\circ} \mathrm{C}$. The zone of inhibition was measured by testing the diameter of inhibition part round each circle.

\section{RESULT AND DISCUSSION}

\section{Morphology of electrospun nanofiber}

The morphology and structure of different nanofibrous mats containing CS/PEO, CS/PEO/Cys, CS/ $\mathrm{PEO} /$ Drugs and $\mathrm{CS} / \mathrm{PEO} / \mathrm{Cys} /$ Drugs were investigated by scanning electron microscopy (SEM). Before the analysis, the samples were dried and coated with a gold layer for 12 hours. Freshly prepared fibers were used to obtain SEM images. Figure 1 presents the fibers $(\mathrm{A}-\mathrm{H})$.

\section{SEM micrograph of CS/PEO}

Figure $1(\mathrm{~A}, \mathrm{~B})$ displays various magnifications of the CS/PEO nanofibers of SEM micrographs. The surface of the fiber is soft and seems almost round. The average diameter was approximately $242 \mathrm{~nm}$. It should be noted that the diameter ranged from $80-500$, which is relatively wide-ranging. During the electrospinning process, since $\mathrm{CS}$ solution is a polycation one, the interaction between the electron donates and receiver causes the jet to breakdown for creating fine nanofibers.

\section{SEM micrograph of CS/PEO/ Cys}

Figure $1(\mathrm{C}, \mathrm{D})$ shows various magnifications of nanofibers and the histogram of fiber diameter distribution. The average diameter was approximately $200 \mathrm{~nm}$. The addition of Cys, a mucoadhesive agent, resulted in decrease of fiber size. Cys is an amino acid type two with polar and nonionic side chains capable of forming hydrogen bond, leading to increasing water solubility. The ionic characteristic efficiency depends on $\mathrm{pH}$.

\section{SEM micrograph of CS/PEO/Drugs}

After addition of the drugs into the polymeric solution, the fiber diameter was reduced to $118 \mathrm{~nm}$, and a uniform distribution of fiber diameter was obtained. (Figure $1(\mathrm{E}, \mathrm{F})$.)

\section{SEM micrograph of CS/PEO/Cyc/Drugs}

Finally, the nanofibrous mat size was approximately $116 \mathrm{~nm}$, and a normal distribution was achieved. Bead formation and spider-like network events were obviously observed in the SEM micrographs (Figure $1(\mathrm{G}, \mathrm{H})$ ).

Figure 2 summarizes type of nanofibers and their sizes. 

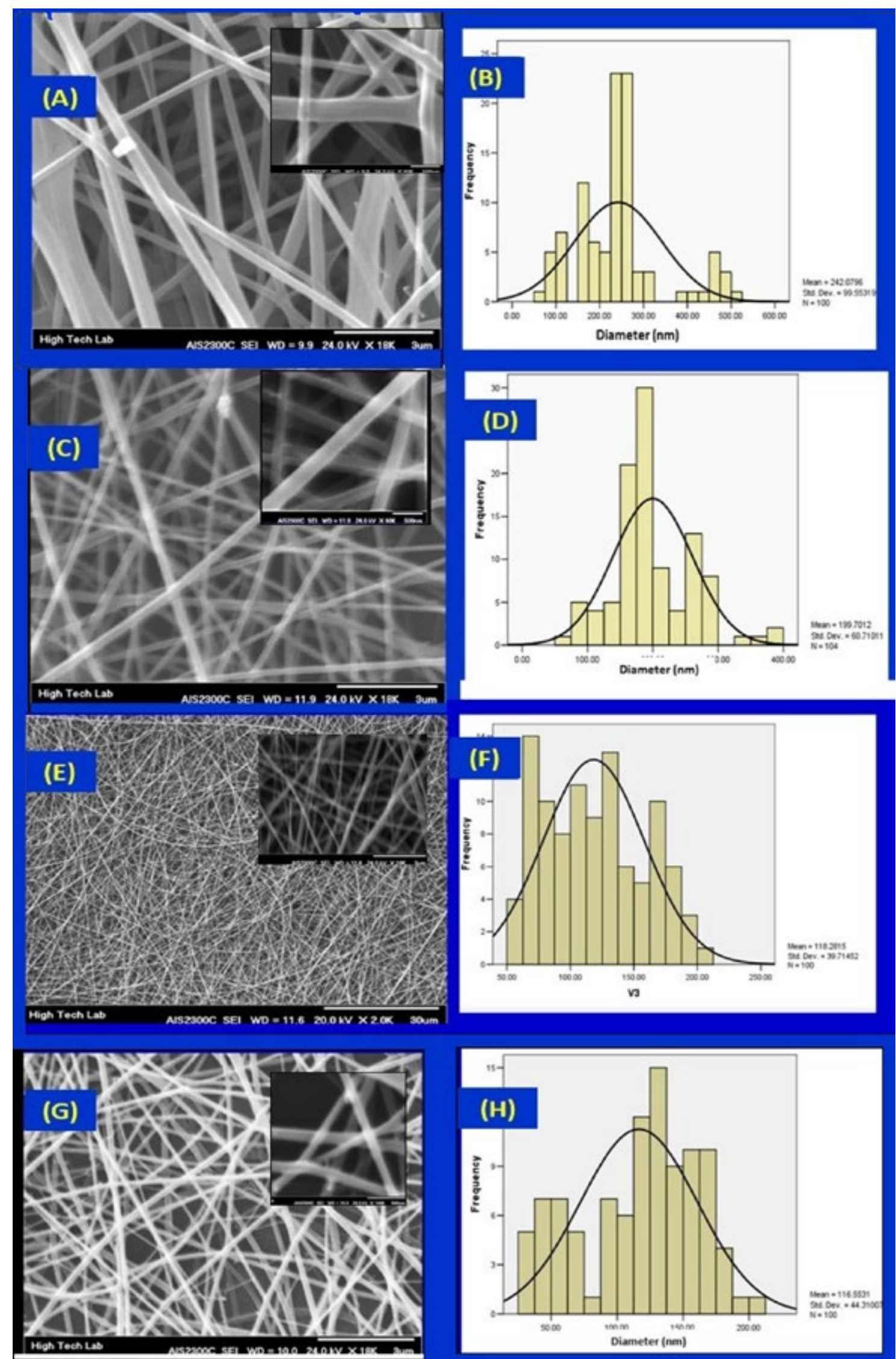

FIGURE 1 - SEM micrographs of (A, B) CS/PEO, (C, D) CS/PEO/Cys, (E, F) CS/PEO/Drugs and, (G, H) CS/ PEO/Cys/Drugs. 


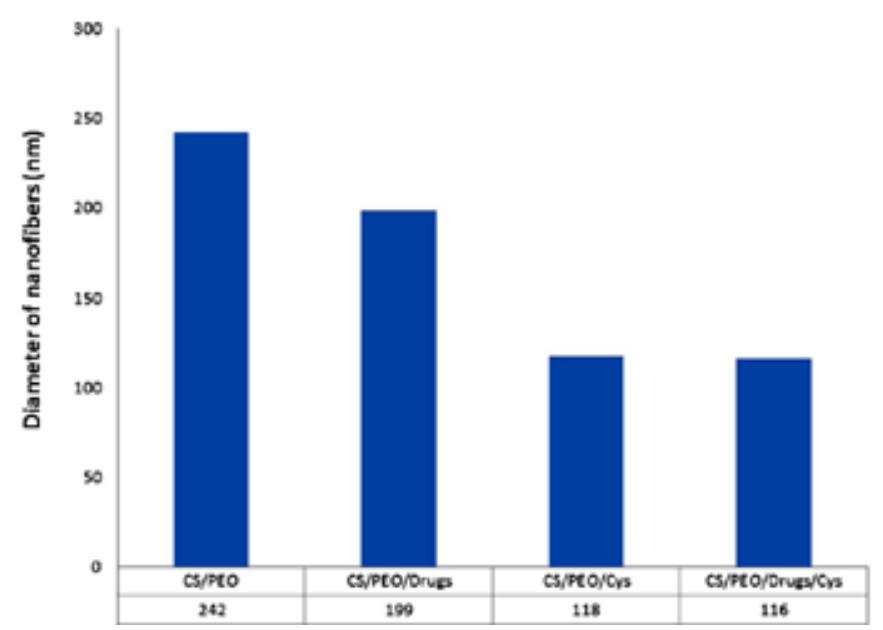

FIGURE 2 - Comparison the diameter of the fibers.

\section{Characterization of nanofibers}

The fibers were characterized by using Fourier Transform Infrared spectroscopy (FT-IR). To measure the FT-IR spectrum of nanoparticles, $2 \mathrm{mg}$ of the samples were mixed with $10 \mathrm{mg} \mathrm{KBr}$ and compressed into a tablet form. The IR spectra of these tablets were obtained in a transition mode and in the spectral region of 400 to $4000 \mathrm{~cm}^{-1}$. As Figure 3 shows, the IR spectra of samples were recorded in a transition mode and in the spectral region of 450 to $4000 \mathrm{~cm}^{-1}$. The peaks of 3359, 2850, $1593 \mathrm{~cm}^{-1}$ belong to $-\mathrm{COOH}, \mathrm{C}-\mathrm{H}$ and $-\mathrm{NH}_{2}$ groups of chitosan (Chen et al., 2001). The peaks at 1658, 1500,1235, 1126 and 1064 $\mathrm{cm}^{-1}$ belong to $\mathrm{C}=\mathrm{C}$, carbonyl, $\mathrm{C}-\mathrm{O}-\mathrm{C}$ and $-\mathrm{C}-\mathrm{N}$ groups of VAN and AMB (Moreno \& Salgado, 2012). The presence of weak spectra in the range of 400 to $500 \mathrm{~cm}^{-1}$ indicates the-S-H groups of Cys (Rath et al., 1994).

In the IR spectrum of CS/PEO, different peaks at $3359,2850,3294 \mathrm{~cm}^{-1}$ shows carboxylic $(-\mathrm{COOH}), \mathrm{C}-\mathrm{H}$, type two amide $(\mathrm{N}-\mathrm{H}, \mathrm{C}-\mathrm{H})$ respectively. Furthermore, a strong peak at $1680 \mathrm{~cm}^{-1}$, a shoulder at $1630 \mathrm{~cm}^{-1}$ and a peak at $1538 \mathrm{~cm}^{-1}$ correspond to $\mathrm{C}=\mathrm{O}$ stretching of the $\mathrm{N}$-acetyl group, scissor vibration of the amine group and ammonium $\left(-\mathrm{NH}^{3+}\right)$ ions, respectively. The peak at 1049 $\mathrm{cm}^{-1}$ is interpreted type one amine groups $\left(\mathrm{R}-\mathrm{NH}_{2}\right)$.

$\mathrm{CS} / \mathrm{PEO} / \mathrm{VANCO}$ spectrum exhibits $\mathrm{C}=\mathrm{C}$ frequency at $1658.7 \mathrm{~cm}^{-1}$ and $\mathrm{C}=\mathrm{O}$ at $1500.62 \mathrm{~cm}^{-1}$. The asymmetric C-O-C absorption occurs at approximately $1235 \mathrm{~cm}^{-1}$. C-N stretching bond was observed at 1260 and $1064 \mathrm{~cm}^{-1}$. C-O stretching vibration is observed in the range of $900-1350$ as a broad peak, by the presence of this bond simple aliphatic ethers can be distinguished. Finally, $\mathrm{C}-\mathrm{Cl}$ stretch in VAN structure $\left(\mathrm{C}_{66} \mathrm{H}_{75} \mathrm{Cl}_{2} \mathrm{~N}_{9} \mathrm{O}_{24}\right)$ appears at approximately 553 $\mathrm{cm}^{-1}$. No peaks belong to undesirable interaction of drug and CS/PEO is detected.
In the IR spectrum of $\mathrm{CS} / \mathrm{PEO} / \mathrm{AMB}$, the bonds of $\mathrm{CS}$ and $\mathrm{PEO}$ are identified, and no unwanted reactions are found. The final CS/PEO solution, including the drugs and Cys, showed all the related peaks.

The weak peaks in the range of 400 to $500 \mathrm{~cm}^{-1}$ confirm the $-\mathrm{S}-\mathrm{H}$ groups of Cys.
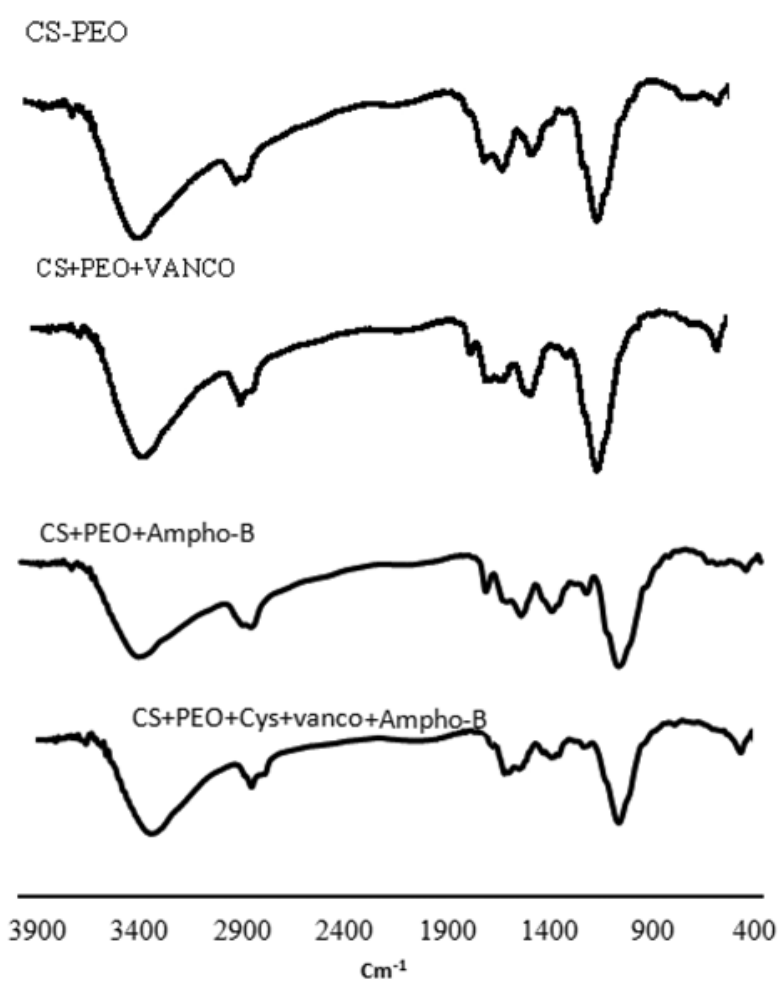

FIGURE 3 - FTIR spectra for CS/PEO, CS/PEO/Cys, CS/PEO/ Drugs, CS/ PEO/Cys/ drugs.

For Differential Scanning Calorimetry (DSC) assay, the samples were sealed in aluminum pans under a nitrogen air atmosphere at a flow rate of $50 \mathrm{~mL} / \mathrm{min}$ and evaluated in $0^{\circ} \mathrm{C}-300^{\circ} \mathrm{C}$ temperature ranges. Figure 4 shows DSC thermograms for CS/PEO, CS/PEO/Cys, and CS/PEO/ drugs. The polymeric fibers showed a melting process with an onset temperature of $52^{\circ} \mathrm{C}$ and a peak of $60^{\circ} \mathrm{C}$ that might be related to the water evaporated from polymers structures. The chitosan and polyethylene oxide showed the exothermic peak at the temperature of $173{ }^{\circ} \mathrm{C}$ and $232^{\circ} \mathrm{C}$, respectively, which are due to the polymer decomposition. The peak temperatures are confirmed in solutions containing CS/ $\mathrm{PEO} / \mathrm{Cys}$, and $\mathrm{CS} / \mathrm{PEO} /$ drugs. However, no melting process was observed for drugs (the melting peak of the drug must be shown in $215^{\circ} \mathrm{C}$ in the fibers) (Kiondo et al., 2011). This phenomenon suggested that there was no crystalline form of the drugs in the fibers (Scapino, 1967).

Dynamical Mechanical Analysis (DMA) of CS/ $\mathrm{PEO}, \mathrm{CS} / \mathrm{PEO} / \mathrm{Cys}$, and $\mathrm{CS} / \mathrm{PEO} /$ drugs nanofibers was 


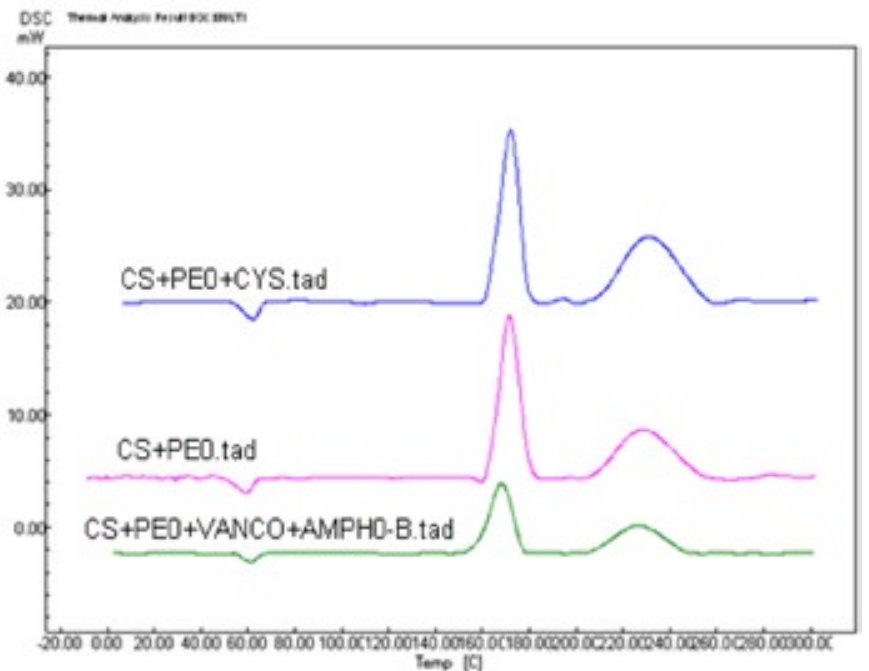

FIGURE 4 - DSC thermograms of CS/PEO, CS/PEO/Cys, CS/ PEO/Cys/ Drugs.

conducted under stress control mode by using STM 50 instrument. The capabilities of the nanofibres to store energy during deformation are expressed in terms of storage modulus, representing the elastic component of a material. As Figure 5 shows, by increasing Cys or drug(s) to the fibers, the modulus value decreased. The fibers with the drug have modules of around $5000 \mathrm{Mpa}$. Fiber with this elasticity has a suitable property for mucosal therapy (Wong, Baji, Leng, 2008).

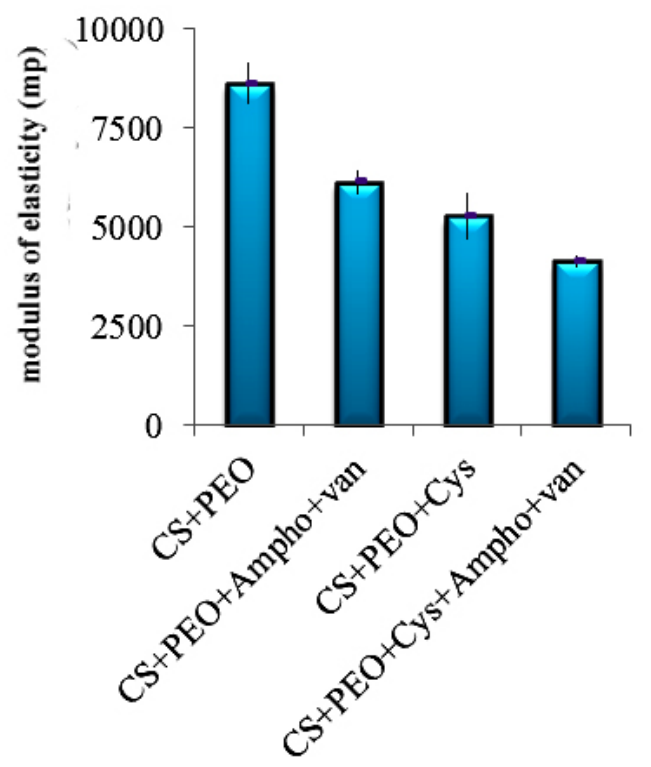

FIGURE 5 - DMA analysis of free and drug-loaded nanofibers.

Also, for mechanical analysis, the tearing resistance was showed in Figure 6 for CS/PEO, CS/PEO/Cys, CS/ $\mathrm{PEO} /$ drugs and $\mathrm{CS} / \mathrm{PEO} / \mathrm{Cys} /$ drugs, respectively. The elongation characteristic of fiber increased with increase in drug concentration that is due to the molecular properties of the drug in comparison to polymers. With increasing the drugs and Cys, fiber diameter reduced approximately $50 \%$ that led to interweaving fibers; therefore, the mechanical property improved (Schmitz et al., 2008).

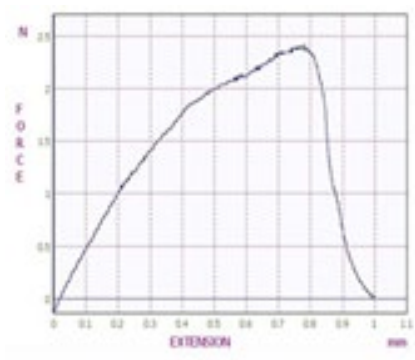

CS-PEO

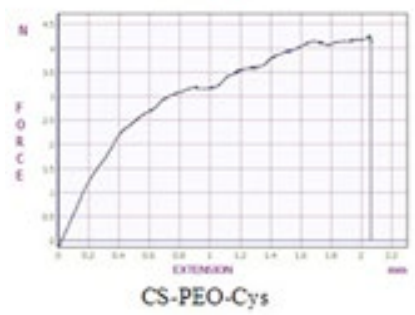

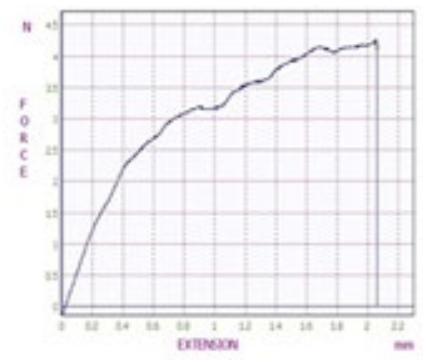

CS.PEO-Drug

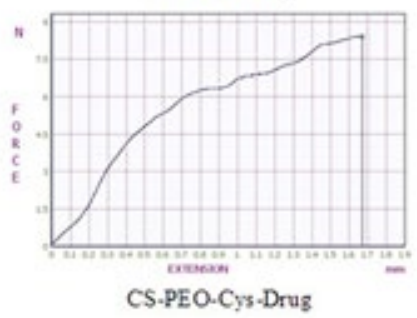

FIGURE 6 - Mechanical analysis of CS/PEO, CS/PEO/Cys, $\mathrm{CS} / \mathrm{PEO} /$ drugs and $\mathrm{CS} / \mathrm{PEO} /$ Cys-drugs fibers.

\section{Mucoadhesive properties}

Mucoadhesion studies were conducted according to the rotating cylinder method. For this propose, mucoadhesive strength of the nanofibers to the oral mucosa was investigated in adult sheep $\left(\mathrm{pH}=6\right.$, at $\left.37^{\circ} \mathrm{C}\right)$. Various concentrations of Cys solution containing (1, 2.5 and $5 \% \mathrm{~W} / \mathrm{W}$ of polymers) were used. The hydration of nanofibers had an important influence on the mucoadhesive strength. In the absence of proper hydration and excessive hydration, mucoadhesive property considerably reduced. Figure 7 shows the relation between Cys concentration and adhesive strength (Data are obtained using at least three experiments \pm standard deviation) (Jia, 2005).

\section{In vitro release and kinetic studies}

Figure 8 exhibits the in vitro release profile of the drugs under sink conditions (PBS, $\mathrm{pH}=7.4$ ). The concentration of the drugs was investigated by the HPLC method. The AMB released in the sustained form was continued to $100 \%$ for 24 hours and VAN had a fast release in the first two hours and was followed by $80 \%$.

The fast release of the drug is highly important in mucoadhesive drug delivery systems, since it increases the patient's compliance. The size of nanofiber has a 


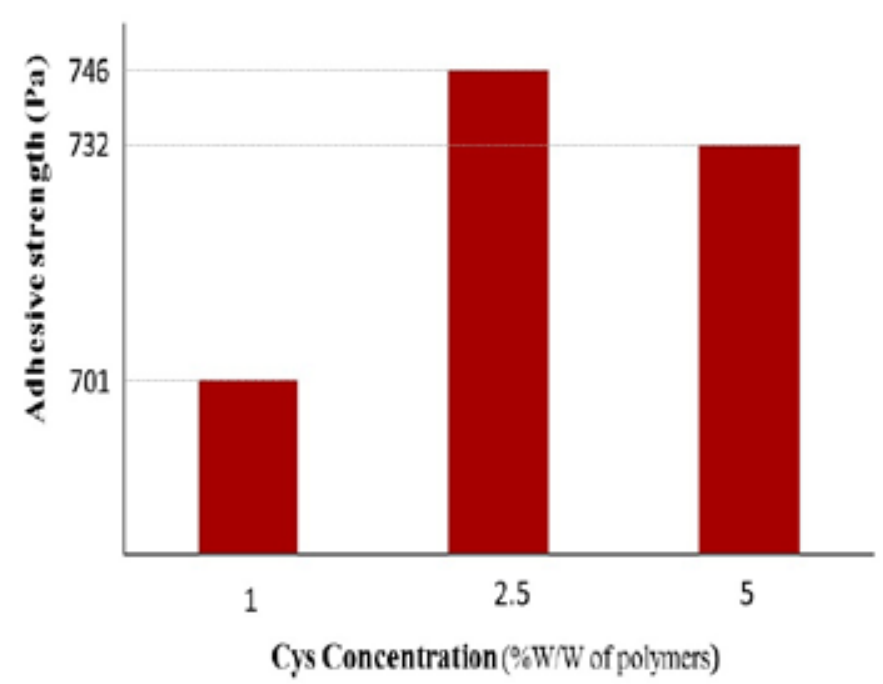

FIGURE 7 - Adhesion strength of nanofibrous mats versus various concentrations of Cys.

considerable effect on the release rate of drugs. Since a fiber with lower diameter often has higher drug release ratio, this behavior is explained by a corresponding increase in surface area to volume ratio. The release behavior of amphotericin B in $24 \mathrm{~h}$ was slow and continuous, leading to a uniform entrance of drug in blood circle and increasing its bioavailability (Schmitz et al., 2008). VAN shows a fast release relative to AMB. It could be due to phase separation transferred to the surface of the fiber or accumulation of VAN molecules as larger units on the surface of fibers. The slow and continuous release of AMB could be due to compatibility of the polymers molecule with the drug.

The kinetic of the drugs was evaluated by the data (curve) fitting method. Various methods of drug release such as zero-order, first-order, Higuchi and HixsonCrowell kinetics were tested, then the most appropriate one was selected based on the regression value $\left(R^{2}\right)$. Table II reports the $R^{2}$ and dissolution rate constant $(\mathrm{K})$ of various kinetic models. Releasing of VAN behaves according to Higuchi, while AMB is followed zero-order equations.

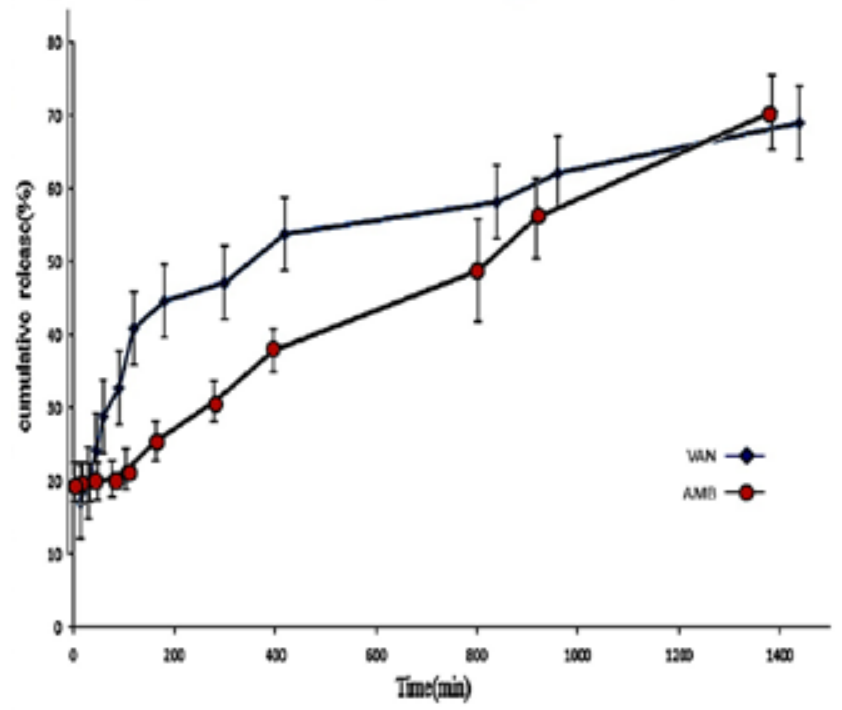

FIGURE 8 - In vitro release profile of VAN and AMB.

\section{Antibacterial and antifungal activity of nanofibers}

The gram-positive streptococcus and candida albicans microorganisms were used for antimicrobial tests. The bacteria and fungus were incubated for the growth of microorganisms on the Muller Hilton and Sabouraud Dextrose agar, respectively. Then, the in vitro antibacterial activities of CS/PEO/Cys, CS/PEO/Vanco, $\mathrm{CS} / \mathrm{PEO} / \mathrm{Cys} /$ Vanco nanofibers and antifungal activities of $\mathrm{CS} / \mathrm{PEO} / \mathrm{Cys}, \mathrm{CS} / \mathrm{PEO} / \mathrm{AMB}, \mathrm{CS} / \mathrm{PEO} / \mathrm{Cys} / \mathrm{AMB}$ nanofibers were examined by the disk diffusion method for 24 hours at $37^{\circ} \mathrm{C}$. The growth of microorganisms was inhibited owing to the antibiotics released in the solution. Figure 9 shows the antibacterial and anti-fungal effects of the fibers.

The diameter of the inhibition zone, showing the diffusion ability of the drugs in the culture, was measured by a ruler (accuracy $=1 \mathrm{~mm}$ ), which is reported in Table III.

Nanofiber without drugs as a control case, has no antibacterial or antifungal effects. Whereas CS/PEO/AMB nanofibers show the best inhibition efficiency.

TABLE II - The $\mathrm{R}^{2}$ and release rate constant values from in vitro release kinetics

\begin{tabular}{llllll}
\hline Formulas & & Zero order & First order & Higuchi model & Hixon \\
\hline AMB loaded Nanofibers & $\mathrm{K}$ & 0.0006 & 0.0017 & 42.873 & 0.0004 \\
& $\mathrm{R}^{2}$ & 0.991 & 0.906 & 0.954 & 0.964 \\
\hline VAN loaded Nanofibers & $\mathrm{K}$ & 0.0003 & 0.006 & 62.67 & 0.002 \\
& $\mathrm{R}^{2}$ & 0.776 & 0.872 & 0.915 & 0.842 \\
\hline
\end{tabular}

$\mathrm{R}^{2}$ : regression coefficient and $\mathrm{k}$ : dissolution rate constant 
TABLE III - The diameter of the zone inhabitation surrounding the fibers

\begin{tabular}{lccccc}
\hline NO. & CS + PEO + Cys & CS+PEO+Cys + Van & CS+PEO+Van & CS+PEO+Ampho & CS+PEO+Cys+Ampho \\
\hline Mean \pm STD & 0 & $57 \pm 0.816 \mathrm{~mm}$ & $60 \pm 0.943 \mathrm{~mm}$ & $9 \pm 0.471$ & $10 \pm 0.474$ \\
\hline
\end{tabular}
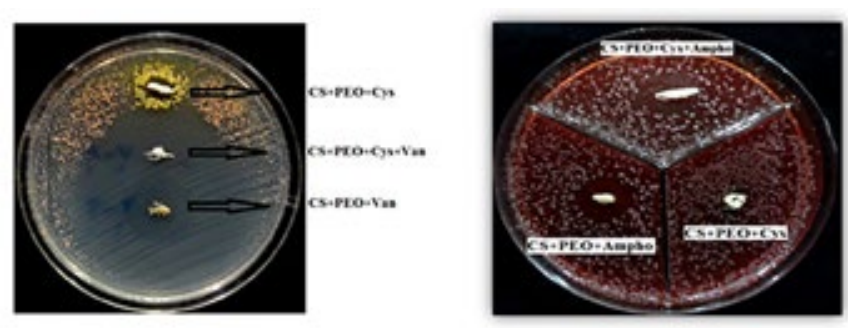

FIGURE 9 - The antibacterial and anti-fungal effect of the drugloaded fibers.

\section{CONCLUSION}

In the present study, new chitosan-based mucoadhesive nanofiber mats were proposed for the RAS test. The mats were simultaneously loaded by VAN and AMB as antibacterial and antifungal agents. Thus, the VAN and AMB were used simultaneously. The SEM images displayed a slight decrease in the fiber diameter with adding drugs and mucoadhesive agents. FTIR spectra confirmed that no undesirable reaction were observed between VAN-AMB and CS-PEO. The DSC test recognized the uniform distribution of drugs in the polymeric bead of the fiber without any crystal form. The elasticity modulus of the nanofiber was in an acceptable range for oral mucosa (about $5 \mathrm{Mpa}$ ). In vitro release profile of VAN was fitted by Higuchi model, whereas AMB was consistent with zero-order equations. The result showed that biodegradable mucoadhesive nanofibrous membranes released high concentrations of VAN in the first 24 hours, but the AMB release was affected in more controlled phenomena. The bioactivity of vancomycin ranged from $90 \%$ to $95 \%$. The antibacterial and antifungal experiments proved the efficiency of nanofibers. Moreover, the results indicated that the nanofibers were functionally active in the treatment of oral aphthous ulceration.

\section{ACKNOWLEDGMENT}

The authors gratefully acknowledge the research council of Kermanshah University of Medical Sciences (Grant Number. 95104) for the financial support.

\section{REFERENCES}

Brooks GF, Jawetz E, Melnick JL, Adelberg EA, Karen CC, Butel JS, et al. Medical microbiology. New York: McGraw-Hill Medical; 2013.

Cannon J, Long M. Emulsions, microemulsions, and lipid-based drug delivery systems for drug solubilization and delivery-part II. In: Rong L (editor). Water-Insoluble Drug Formulation, 2nd ed. CRC Press; 2008. p. 227-54.

Chen X, Knight DP, Shao Z, Vollrath F. Regenerated Bombyx silk solutions studied with rheometry and FTIR. Polymer. 2001;42(25):09969-74.

Chiu H, Tsai T. Topical use of systemic drugs in dermatology: A comprehensive review. JAAD. 2011;65(5):22-30.

Costa-Pinto AR, Reis R L, Neves N M. Scaffolds based bone tissue engineering: the role of chitosan. Tissue Eng Part B Rev. 2011;17(5):331-47.

Fullana MJ, Wnek GE. Electrospun collagen and its applications in regenerative medicine. Drug Deliv Transl Res. 2012;2(5):31322.

Hamill RJ. Amphotericin B formulations: a comparative review of efficacy and toxicity. Drugs. 2013;73(9):919-34.

Hargreaves PL, Nguyen T, Ryan RO. Spectroscopic studies of amphotericin B solubilized in nanoscale bilayer membranes. BBA-Bioenergetics. 2006;1758(1):38-44.

Jia L. Nanoparticle formulation increases oral bioavailability of poorly soluble drugs: approaches, experimental evidence and theory. Curr Nanosci. 2005;1(3):237-43.

Kiondo P, Wamuyu-maina G, Bimenya GS, Tumwesigye NM, Wandabwa J, Okong P. Risk factors for pre-eclampsia in Mulago Hospital, Kampala, Uganda. Am J Trop Med Hyg. 2011;17(4):480-7. 
Lu H, Oh HH, Kawazoe N, Yamagishi K, Chen G. PLLAcollagen and PLLA-gelatin hybrid scaffolds with the funnel-like porous structure for skin tissue engineering. Sci Tech Adv Mater. 2012;13(6):064210-8.

Li D, Xia Y. Electrospinning of nanofibers: reinventing the wheel? Adv Mater. 2004;16(14):1151-70.

Ma L, Deng L, Chen J. Applications of poly (ethylene oxide) in controlled release tablet systems: a review. Drug Dev Ind Pharm. 2013;40(7):845-51.

Ma Z, Kotaki M, Inai R, Ramakrishna S. Potential of nanofiber matrix as tissue-engineering scaffolds. J Tissue Eng. 2005;11(12):101-9.

Moreno AD, Salgado HR. Development and validation of the quantitative analysis of ceftazidime in powder for injection by infrared spectroscopy. Phys Chem. 2012;2(1):6-11.

Passaretti D, Silverman RP, Huang W, Kirchhoff CH, Ashiku S, Randolph MA, et al. Cultured chondrocytes produce injectable tissue-engineered cartilage in hydrogel polymer. J Tissue Eng. 2001;7(6):805-15.

Puppi D, Chiellini F, Piras A, Chiellini E. Polymeric materials for bone and cartilage repair. Prog Polym. Sci. 2010;35(4):40340.
Rostami E, Kashanian S, Azandaryani A. Preparation of solid lipid nanoparticles as drug carriers for levothyroxine sodium with in vitro drug delivery kinetic characterization. Mol Biol Rep. 2014;41(5):3521-7.

Rath P, Bovee-geurts P, Degrip W, Rothschild K. Photoactivation of rhodopsin involves alterations in cysteine side chains: Detection of an S-H band in the Meta I--Meta II FTIR difference spectrum. Biophys J. 1994;66(6):2085-91.

Scapino RP. Biomechanics of prehensile oral mucosa. J Morphol Embryol. 1967;122(2):89-114.

Schmitz T, Grabovac V, Palmberger TF, Hoffer MH, Bernkopschnãrch A. Synthesis and characterization of a Chitosan-Nacetyl cysteine conjugate. Int J Pharm. 2008;347(1-2):79-85.

Venkateswarlu V, Manjunath K. Preparation, characterization and in vitro release kinetics of clozapine solid lipid nanoparticles. J Control Release. 2004;95(3):627-38.

Wong S, Baji A, Leng S. Effect of fiber diameter on tensile properties of electrospun poly( $\varepsilon$-caprolactone). Polymer. 2008;49(21):4713-22.

Received for publication on 08th June 2017 Accepted for publication on 24th April 2018 